\title{
Sesearch Square
Identification of neoadjuvant radiotherapy \\ resistance genes in rectal cancer using integrated bioinformatics analysis
}

\section{Caihua Liao}

Guilin Medical University

\section{Tiancheng Zhang}

Key Lab of Reproduction of the National Population and Family Planning Commission-Shanghai Institute of Planned Parenthood Research,Fudan University Reprodution and Development Institution

\section{Ke Wang}

Guilin Medical University

Qiong Zhong

Guilin Medical University

\section{Lin Chen}

Shanghai East Hospital

\section{Haiyan Dong}

Key Lab of Reproduction Regulation of the National Population and Family Planning CommissionShanghai Institute of Planned Parenthood Research,Fudan University Reproduction and Development Institution

\section{Chuangang Fu}

Shanghai East Hospital

Rongzhong Jin ( $\nabla$ rongzhong_jin@163.com )

Guilin Medical University

\section{Research article}

Keywords: neoadjuvant radiotherapy resistance, rectal cancer, microarray, functional enrichment, chemokines

Posted Date: March 10th, 2020

DOI: https://doi.org/10.21203/rs.3.rs-16673/v1

License: (c) (i) This work is licensed under a Creative Commons Attribution 4.0 International License. Read Full License 


\section{Abstract}

The incidence of rectal cancer is increasing year by year. And most patients have been diagnosed in the advanced stages largely because of the special anatomical location of the rectum, resulting in uneasy diagnosis in its early stage. The combination of radiotherapy and chemotherapy is widely accepted as effective treatment to the rectal cancer, significantly reduce the local recurrence rate and improve longterm survival, but the occurrence of radiotherapy resistance of rectal cancer strikingly weaken the responding of treatment. So finding reasons or factors leading to the resistance of neoadjuvant radiotherapy for rectal cancer become very urgent not just for the current clinic but also for development of new therapeutical approaches. To identify potential biomarkers associated with neoadjuvant radiotherapy resistance in rectal cancer using bioinformatics analysis. Firstly, we extracted relevant data from GEO database and analyzed the corresponding data by a series of analytic methods. Then, the candidate genes were identified by performing of the analysis of protein-protein interaction network, KEGG and GO, subsequently comfirmed at the levels of mRNA and Protein with Oncomine and The Human Protein Atlas respectively. Furthermore, these comfirmed genes were submitted to CMap analysis to identify candidate therapeutic compounds for neoadjuvant radiotherapy resistance. We found that the abnormal expression of some chemokines were tightly associated with the rectal cancer neoadjuvant radiotherapy resistance. Especially the upreuglation of Chemokines 9(CXCL9) and Chemokines 10(CXCL10) and downregulation of Chemokines 13(CXCL13) and Chemokines 5(CCL5) might be the potential biomarks of the radiotherapy resistance of rectal cancer. Through a series of analyses, the molecules that may be related to the radiotherapy resistance of rectal cancer were identified that may have a hint effect on the treatment of radiotherapy resistance of rectal cancer.

\section{Introduction}

The advantages of preoperative neoadjuvant radiotherapy include a good blood supply of target cells, high sensitivity to radiation, and efficacy. Radiotherapy can shrink tumors, leading to significantly improving the curative tumor resection and anal preservation rates [1, 2]. Therefore, neoadjuvant radiotherapy has become the standard of care for patients with resectable rectal cancer. But, because of radiotherapy resistance, a considerable proportion of patients were unable to be benefit from neoadjuvant radiotherapy. Hence, the clinical response of neoadjuvant radiotherapy needs further evaluation with respect to resistance genes and mechanisms in order to help physicians prepare more effective therapeutic approaches.

Until recently, some progresses were obtained in the field of neoadjuvant radiotherapy resistance. For example, MiR-17-5p has been reported to significantly enhance the sensitivity of X-rays to radioresistant cells as well as downregulate and promote antiradioactive phenotypes in esophageal adenocarcinoma cancer stem-like cells [3]. Overexpression of TCN1 is reportedly associated with adverse treatment responses and outcomes in rectal cancer patients receiving concurrent neoadjuvant chemoradiotherapy, indicating the potential prognostic value of this gene [4]. Elevated levels of $\beta$-catenin have been shown to inhibit proliferation of colorectal cancer cells in vitro and growth of mouse xenograft tumors [5], and 
heightened expression of Rhoa has been shown to be a good predictor of poor prognosis due to high treatment resistance rates [6]. Nonetheless, specific mechanism(s) associated with newly developed radiation resistance are not fully understood as it is a complex process involving a large number of genes.

Rapid development of high-throughput biotechnologies, such as microarray, has made it possible to analyze RNA expression profiles, thereby enhancing our understanding of various diseases and their molecular mechanisms [7-9]. However, few studies have systematically analyzed gene expression profiles of neoadjuvant radiotherapy resistance by integrating bioinformatics analysis. In the present study, neoadjuvant radiotherapy resistance gene expression datasets were obtained from Gene Expression Omnibus and systematic bioinformatics analysis performed, including identification of differentially expressed genes (DEGs), functional enrichment and co-expression network analyses, and identification of significantly differently expressed biomarkers of neoadjuvant radiotherapy resistance in colorectal cancer. The results of the present study will significantly improve our understanding of radiotherapy resistance mechanisms and efficacy.

\section{Results}

\section{Identification of DEGs}

The GSE60331 date set was obtained from the Gene Expression Omnibus database. Analysis of radiotherapy-resistant group (8 radiotherapy-resistant rectal tumor group, 8 radiotherapy-resistant rectal normal group samples), and radiotherapy-sensitive group (6 radiotherapy-sensitive rectal tumor group, 6 radiotherapy-sensitive rectal normal group) obtained 1325 and 1247 related genes, respectively. A total of 1000 DEGs were common to the radiotherapy-resistant and radiotherapy-sensitive groups. These DEGs were thought to be common in rectal cancer and related to its occurrence, but not necessarily all specifically related to neoadjuvant radiotherapy resistance, so we ignore these genes. In the radiotherapyresistant group, we found 325 unique DEGs thought to be related to neoadjuvant radiotherapy resistance in rectal cancer and 247 in the radiotherapy-sensitive group (Fig. 1A). Genes with corrected $P<0.05$ and an absolute fold change $>2$ were considered as DEGs after the original data was corrected by Limma (Fig. 1B). The specific resistance genes founded in the radiotherapy-resistant group showed that normal and cancerous tissues could be separated by cluster analysis(Fig. 1C); the left 8 samples were in the radiotherapy-resistant rectal tumor group, while the right 8 samples were in the radiotherapy-resistant rectal normal group. This result confirmed the reliability of the analysis. Based on this method, we found that 325 genes between the neoadjuvant radiotherapy-resistant and radiotherapy-sensitive groups were significantly differentially expressed [169 up-regulated, 156 downregulated] (Fig. 1D).

\section{KEGG and GO Enrichment}

To get deeper information about DEG function, GO enrichment on the above 325 DEGs was carried out with the aid of the Database for Annotation, Visualization, and Integrated Discovery with a $P<0.05$. GO 
results showed that 20 pathways were enriched (Fig. 2A), mainly including metabolic pathways; cellular response to zinc ion, negative regulation of growth, cellular response to cadmium ion, drug transport, endonucleolytic cleavage in ITS1 to separate SSU-rRNA from 5.8S rRNA and LSU-rRNA from tricistronic rRNA transcript (SSU-rRNA, 5.8S rRNA, LSU-rRNA), proteolysis, positive regulation of osteoblast proliferation, cytoskeletal anchoring at plasma membrane, magnesium ion transport, digestion, complement activation, classical pathway, magnesium ion transmembrane transport, chemokinemediated signaling pathway, D-amino acid metabolic process, folic acid metabolic process, drug transmembrane transport, muscle cell cellular homeostasis, error-prone translesion synthesis, neuron projection morphogenesis, proteolysis involved in cellular protein catabolic process, extracellular exosome, peroxisomal matrix, peroxisome, extracellular space, cell, external side of plasma membrane, photoreceptor outer segment membrane, extracellular region, CXCR3 chemokine receptor binding, receptor binding, antigen binding, chemokine activity, PDZ domain binding, electron carrier activity, cofactor binding, magnesium ion transmembrane transporter activity, D-amino-acid oxidase activity, receptor signaling protein tyrosine kinase activator activity, 1-alkylglycerophosphocholine 0acetyltransferase activity. The top 10 results of the $\mathrm{GO}$ enrichment analysis are shown in Table 1. Moreover, we also found DEGs enriched in different GO terms. Twenty pathways were enriched in biological processes, 8 were enriched in cellular components, and 11 were enriched in molecular unction $(P<0.05)$. Then we classify these $G O$ terms according to biological processes, cell component and molecular functions, and shown in Fig. 2B.

KEGG pathway enrichment was also carried out on the above 325 DEGs using the KEGG Automatic Annotation Server (http://www.genome.jp/tools/kaas/). The results showed enrichment of 20 pathways (Fig. 2C), with DEGs mainly enriched in metabolic pathways; mineral absorption; peroxisomes; purine metabolism; chemokine signaling pathways; pyrimidine metabolism; glycine, serine, and threonine metabolism; fatty acid degradation; Wnt signaling pathways; fatty acid metabolism; steroid hormone biosynthesis; long-term depression; DNA replication; alanine, aspartate, and glutamate metabolism; lysosomes; base excision repair; insulin secretion; cytokine-cytokine receptor interactions; protein digestion and absorption; and nucleotide excision repair. The top 10 results of the KEGG enrichment analysis are shown in Table 2.

\section{PPI network construction and analysis}

The PPI relationships between DEGs were determined using the Search Tool for the Retrieval of Interacting Genes/Proteins, then a PPI network was constructed with Cytoscape. Finally, a network with 51 nodes and 116 edges was built (Fig. 3A). The top 18 degree hub nodes in the PPI network were DCAF13, BRIX1, NMU, RRS1, CIRH1A, PN01, CXCL10, CXCL9, CCL5, NPY, POLRIE, DDX27, RCL1, GRM8, CXCL13, BMP2, DPP4, and POLR1D. These genes/proteins might play an important role in neoadjuvant radiotherapy resistance of rectal cancer (Fig. 3B). Importantly, 4 genes (CXCL9, CXCL10, CXCL13, and CCL5) were found to be enriched in each analysis. Therefore, it was postulated that these 4 genes were closely related to neoadjuvant radiotherapy resistance. 


\section{Key gene cross analysis and validation}

Further analysis of KEGG pathway enrichment and PPI network data revealed that DEGs CXCL9 and CXCL10 were highly expressed, while CXCL13 and CCL5 had low expression in the radiotherapy-resistant rectal tumor group. The immunohistochemical schematic diagrams of CXCL9, CXCL10, CXCL13 and CCL5 genes for rectal cancers and normal rectal tissues were obtained from The Human Protein Atlas. Meanwhile, the mRNA expression of these genes in rectal cancers was analyzed by Oncomine, the obtained results were shown in Fig. 4. The X-axis represented the sample grouping and the $Y$-axis represented expression value in corresponding genes in the right of Fig. 4. The expression of CXCL9 is lower in normal tissues of rectal than that in tumor tissues of ractal (Fig. 4A right). The expression of CXCL10 is high in normal tissues of rectal, but the expression is higher in tumor tissues of ractal (Fig. 4B right). The expression level of CXCL13 is high in normal tissues of colorectum (Fig. $4 \mathrm{C}$ right $\mathrm{X}$ axis label $1-5)$, and X-axis label 6 is tumor tissues of colorectum. However the expression level is low in tumor tissues of colorectum (Fig. $4 \mathrm{C}$ right $X$ axis label 6). The expression level of CCL5 was higher normal tissues of rectum than that in tumor tissues of ractum (Fig. 4D right). The result is that CXCL9 and CXCL10 were highly expressed, while CXCL13 and CCL5 had low expression in colorectal tumor group. So the obtained results of which are consistent with our analysis.

\section{CMap Analysis}

CMap, as a bioinformatic tool, shows functional connections between small agents and gene expression signatures of diseases. In this study, only 215 genes out the obtained 325 DEGs were successfully converted into the probes. And then submitted to CMap for further analysis. The results from the CMAP were analyzed according to their $P$ values, and enrichment scores. As a result we identified top 7 negatively related small molecules drugs were listed in Table 3 and PCMap-value of $<0.0001$ (Table 3 ).

\section{Discussion}

Radiation tolerance is a major factor related to failure of radiotherapy and poor prognosis for cancer patients. Therefore, radiotherapy resistance has become a major obstacle to overcome $[10,11]$. Radiotherapy resistance gene chip analysis of rectal cancer has the advantage of being able to provide a much larger amount of data than other previous biotechnology methods, allowing for more extensive experimental design and analytical scope $[12,13]$. In the present study, an integrated bioinformatics analysis approach was used to identify potential biomarkers of neoadjuvant radiotherapy resistance in rectal cancer. Ultimately, the 4 differentially expressed chemokine genes (CXCL 9, CXCL10, CXL13, and CCL5) were identified as being related to radiotherapy resistance in colorectal cancer.

These results are in agreement with previous studies reporting a close relationship between chemokines and neoadjuvant radiochemotherapeutic efficacy [14]. CXCR4, CXCL12, and CXCR7 have been suggested to be able predict neoadjuvant radiochemotherapy and radiotherapy resistance in $\mathrm{T}$ - and $\mathrm{N}$-state rectal tumors, with high CXCR4 expression indicating a poor prognosis [15]. Moreover, upregulation of CXCL12 
(Sdf-1a) has been shown to induce neoadjuvant radiotherapy resistance [16]. CXCL9, CXCL10, and CXCL11 proteins have also been reported to inhibit the formation of tumor blood vessels through their coligands; hence, overexpression of CXCL9, CXCL10, and CXCL11 can inhibit the growth and metastasis of colorectal cancer [17]. Thus, we concluded that abnormal expression of chemokines maybe the key factors that leading to occurrence of radiotherapy resistance.

Herein, high CXCL9 and CXCL10 expression was found to be related to radiotherapy resistance in rectal cancer cells. This is in agreement with previous studies showing that high CXCL10 expression inhibits growth of certain cancer cells [18], and high CXCL10, CXCL8, and CXCL11 expression lead to a decrease in the survival rate of breast cancer patients [19]. Moreover, overexpression of CXCL9 and CXCL10 has been related to a high degree of tumor invasion [20]. CXCR3directed chemokines CXCL9 and CXCL10 induce Cathepsin B in human breast cancer cells, thereby supporting tumor invasion and metastasis[21].

The results of the present study also predicted that low CXCL13 and CCL5 expression is related to radiotherapy resistance in rectal cancer cells. A previous study reported that transforming growth factor- $\beta$ signaling and Oct4 synergistically induce expression of the epithelial-to-mesenchymal transition-related genes Snail, Slug, and CXCL13, which accelerates disease progression, especially in late stages, and may indicate a poor prognosis in breast cancer patients [22]. Overexpression of CCL 5 has also been shown to lead to migration and proliferation of breast cancer cells [23]. CXCL13 has also been shown to participate in the migration and invasion of androgen-induced prostate cancer due to upregulation by the androgen receptor axis at mRNA and protein levels. Thus, CXCL13 is considered to be an androgen-responsive gene in prostate cancer patients with higher than normal androgen levels [24]. In contrast, PKCE overexpression and PTEN loss have also been reported to cooperatively upregulate production of CXCL13, inhibiting the migration and tumorigenicity of prostate cancer [25]. At the same time, low CXCL13 expression in gastric cancer patients has been associated with a better prognosis [26]. However, blocking the CCR5/CCL5 axis may be prevent metastasis and provide more therapeutic strategies to control the progression of pancreatic cancer [27]. Nevertheless, it is obvious that these chemokines (CXCL9, CXCL10, CXCL13, and CCL5) have certain influences on the occurrence and development of tumors.

Neoadjuvant radiotherapy kills tumor cells through the cytotoxic action of radiation and drugs and its effects on the surrounding microenvironment. The tumor microenvironment is composed of a variety of components, including chemokines, plays an important role in the tumor progression and pathophysiological processes [28]. Chemokine receptors and ligands are abundantly expressed in inflammatory environments and increasing evidence indicates that chemokines are involved in the regulation of tumor proliferation, angiogenesis, apoptosis, and metastasis in cancer-related inflammation and can be used as therapeutic targets. CXCR4 has been reported to be upregulated by the microenvironment in colon cancer, and CXCR4 inhibitors have the potential to inhibit metastatic growth of tumors [29]. CXCR2/CXCL7 have also shown prognostic value in colorectal cancer. Colorectal cancer cells secrete interleukin-8, which mediates the homing of mesenchymal stem cells and induces CXCL7 production. The disease-free and overall survival of patients with high CXCR2/CXCL7 expression in colon 
cancer metastases are both shortened [30]. Moreover, overexpression of CCR6 has been shown to be a key factor in promoting the metastasis of colorectal cancer cells in vivo, especially to the liver [14]. The concentration gradients of CCR6 and CCL20 in colorectal cancer provide a pathway for the specific transfer (metastasis) of colorectal cancer cells to the liver. In breast cancer cells, transfection of CXCL7 stimulates Matrigel invasion and secretion of the lymphangiogenic factors vascular endothelial growth factor- $C$ and $-D[31,32]$. Although the results of our analysis are not completely consistent with the outcomes of the above studies, we suspect this may be due to a decrease in the expression of CXCL13 and CCL5, which leads to changes in the tumor microenvironment. However, such mechanisms of action remain to be explored.

The ability to screen patients with colorectal cancer who are resistant to neoadjuvant radiotherapy is very important to providing alternative treatment regimens and improving prognoses. Although the present results require further in vivo/in vitro validation, it is clear that the effects of chemokines in cancer should be further investigated. Nevertheless, integrated bioinformatics analyses are a very useful tool for pinpointing genes/proteins of interest which could aid the development of new targeted therapies for radiotherapy-resistant colorectal cancer.

\section{Conclusions}

Radiotherapy-resistant is closely related to the failure of radiotherapy and the poor prognosis of cancer patients. Neoadjuvant radiotherapy kills cancer cells through the cytotoxic effects of radiation and drugs and their effects on the surrounding microenvironment. Studies have shown that chemokines can play a role in the development of tumors. Chemokines play an important role in the alteration of tumor microenvironment. Therefore, Therefore, we speculate that chemokines may have a certain effect on radiotherapy for rectal cancer. This will be of some significance for us to explore their molecular mechanisms.

\section{Materials And Methods}

\section{Data sources}

The GSE60331 gene expression profile was obtained from the Gene Expression Omnibus database (http://www.ncbi.nlm.nih.gov/geo/) of the National Center for Biotechnology Information; this platform was applied to expression arrays. Data were analyzed from a total of 28 tissue samples include 8 radiotherapy-resistant rectal tumor group, 8 radiotherapy-resistant rectal normal group, 6 radiotherapysensitive rectal tumor group, 6 radiotherapy-sensitive rectal normal group, the criteria were data from tumor samples and normal tissue; biopsies were taken at two different timepoints: the first time is when tumor and normal mucosa before treatment and the second time is only tumor (week 3 ) that loading dose of bevacizumab but before chemoradiation. CEL forms and annotation files were downloaded for further analysis.

\section{Identification of DEGs}


Microarray data was preprocessed in Bioconductor with the Affy software package [33] of R (version 3.3.2) software. The robust multivariate average algorithm [34] was used for background correction, quartile normalization, and probe summarization to obtain gene expression matrices. The data were normalized using the Limma package of $\mathrm{R}$ software and then by the Bayes method [35]. DEGs between cancerous and normal tissues in radiotherapy-resistant and -sensitive groups were screened separately using a $\mathrm{P} \leq 0.05$ and $\mid \log$ (Fold Change) $\mid>1$ as filter conditions. Then, DEGs in both groups were merge, and the duplicates were removed. Finally, specific DEGs associated with colon cancer radiotherapy resistance or sensitivity were obtained. The original expression datasets under all conditions were normalized with the robust multiarray average method [36] using the ClusterProfiler package in $R$ software to obtain the gene expression matrix. The t-test method in the R software Limma package was used to identify DEGs between radiotherapy-resistant and -sensitive group genes. Values with a llog(Fold Change) $\mid>2.0$ and $P<0.05$ were selected as cutoff criteria.

\section{Functional enrichment analysis}

In order to better understand the functions of the selected DEGs, Gene Ontology [GO] [37] functional and Kyoto Encyclopedia of Genes and Genomes (KEGG) pathway enrichment analyses were performed for radiotherapy-resistant DEGs to identify signaling pathways potentially involved in the development of radiotherapy resistance in rectal cancer. The Database for Annotation, Visualization, and Integrated Discovery (https://david.ncifcrf.gov) was used for GO enrichment of DEGs, and the KEGG Automatic Annotation Server (http://www.genome.jp/tools/kaas/) was used for KEGG pathway enrichment [37, 38] with a $\mathrm{P}<0.05$ and number of genes $>2$. The ggplot2 package in $\mathrm{R}$ software was used to create $\mathrm{GO}$ and KEGG histograms. Then we draw the column diagram through barplot.

\section{Protein co-expression network.}

The Search Tool for the Retrieval of Interacting Genes/Proteins database provided comprehensive interaction information between proteins, including predicted interactions and experimental studies [39]. Cytoscape (version 3.5.1) is a traditional bioinformatics software for visual biology network and data integration. Herein, the Search Tool for the Retrieval of Interacting Genes/Proteins was used to analyze protein-protein interactions (PPI) with integration scores between DEGs and DEGs $>0.4$. Then, the protein co-expression network was built by Cytoscape software [40] .

\section{Analysis of mRNA expression in human rectal cancer}

Related rectal cancer gene expression was identified by analyzing Roessler and TCGA databases available on Oncomine (www.oncomine.org). High and low values were respectively defined as above and below the mean value.

\section{Analysis of protein expression in human rectal cancer}

Protein expression in rectal tumor tissues and normal tissues was determined through the human protein atlas (www.proteinatlas.org) to screen and capture the key proteins that may play important roles in 
radiotherapy resistance of rectal cancer.

\section{Analysis of DEGs by CMap}

The DEGs were converted into probes. These probes were collated into tag sets of "up" or "down" genes, and analyzed using the CMap website (https://portals.broadinstitute.org/cmap/)[41]. The similarity was reported as connectivity score ranging from +1 to -1 . Positive connectivity score denotes increased similarity while negative score denotes inverse similarity between the expression patterns induced by the compounds in the CMap database and the query DEGs.

\section{Declarations}

\section{Acknowledgements}

\section{Authors' contributions}

Caihua Liao, Tiancheng Zhang and Ke Wang selected the data,performed data analysis and drafted the manuscript. Qiong Zhong, Chen Lin and Haiyan Dong contributed to the analysis of the results and revised the manuscript critically for important intellectual content. Chuangang Fu and Rongzhong Jin designed the study as well as the critical review of the manuscript.

\section{Funding}

This work was supported by the Youth Foundation of the Shanghai Institute of Planned Parenthood Research and the Shanghai Municipal Commission of Health and Family Planning (20164Y0267), as well as the National Natural Science Foundation of China (81573004 and 81272561).

\section{CONFLICT OF INTERESTS}

The authors declare that they have no competing interests.

\section{Consent for publication}

Not applicable

\section{Availability of data and materials}

The datasets during and/or analysed during the current study available from the corresponding author on reasonable request.

\section{References}

[1] Roh MS, Colangelo LH, O'Connell MJ, Yothers G, Deutsch M, Allegra CJ, et al. Preoperative multimodality therapy improves disease-free survival in patients with carcinoma of the rectum: NSABP R03. J CLIN ONCOL. 2009;27冈5124-30. 
[2] Li Y, Wang J, Ma X, Tan L, Yan Y, Xue C, et al. A Review of Neoadjuvant Chemoradiotherapy for Locally Advanced Rectal Cancer. INT J BIOL SCI. 2016;12ه1022-31.

[3] Lynam-Lennon N, Heavey S, Sommerville G, Bibby BA, Ffrench B, Quinn J, et al. MicroRNA-17 is downregulated in esophageal adenocarcinoma cancer stem-like cells and promotes a radioresistant phenotype. Oncotarget. 2017;8®11400-13.

[4] Lee YY, Wei YC, Tian YF, Sun DP, Sheu MJ, Yang CC, et al. Overexpression of Transcobalamin 1 is an Independent Negative Prognosticator in Rectal Cancers Receiving Concurrent Chemoradiotherapy. J CANCER. 2017;8ه1330-7.

[5] Annunziata CM, Kohn EC. Novel facts about FAK: new connections to drug resistance? J Natl Cancer Inst. 2013;105®1430-1.

[6] Ruihua H, Mengyi Z, Chong Z, Meng Q, Xin M, Qiulin T, et al. RhoA regulates resistance to irinotecan by regulating membrane transporter and apoptosis signaling in colorectal cancer. Oncotarget. 2016;7区 87136-46.

[7] Li S, Teng S, Xu J, Su G, Zhang Y, Zhao J, et al. Microarray is an efficient tool for circRNA profiling. BRIEF BIOINFORM. 2018.

[8] Schena M, Heller RA, Theriault TP, Konrad K, Lachenmeier E, Davis RW. Microarrays: biotechnology's discovery platform for functional genomics. TRENDS BIOTECHNOL. 1998;16®301-6.

[9] Blencowe BJ, Ahmad S, Lee LJ. Current-generation high-throughput sequencing: deepening insights into mammalian transcriptomes. Genes Dev. 2009;23ه1379-86.

[10] Li F, Zhou K, Gao L, Zhang B, Li W, Yan W, et al. Radiation induces the generation of cancer stem cells: A novel mechanism for cancer radioresistance. ONCOL LETT. 2016;12『3059-65.

[11] Tang L, Wei F, Wu Y, He Y, Shi L, Xiong F, et al. Role of metabolism in cancer cell radioresistance and radiosensitization methods. J Exp Clin Cancer Res. 2018;37ه87.

[12] Kharchenko PV, Tolstorukov MY, Park PJ. Design and analysis of ChIP-seq experiments for DNAbinding proteins. NAT BIOTECHNOL. 2008;26冈1351-9.

[13] Peng S, Alekseyenko AA, Larschan E, Kuroda MI, Park PJ. Normalization and experimental design for ChIP-chip data. BMC BIOINFORMATICS. 2007;8区219.

[14] Liu J, Ke F, Xu Z, Liu Z, Zhang L, Yan S, et al. CCR6 is a prognostic marker for overall survival in patients with colorectal cancer, and its overexpression enhances metastasis in vivo. PLOS ONE. 2014;98 e101137. 
[15] D'Alterio C, Avallone A, Tatangelo F, Delrio P, Pecori B, Cella L, et al. A prognostic model comprising pT stage, $\mathrm{N}$ status, and the chemokine receptors CXCR4 and CXCR7 powerfully predicts outcome in neoadjuvant resistant rectal cancer patients. INT J CANCER. 2014;135®379-90.

[16] Kim HJ, Bae SB, Jeong D, Kim ES, Kim CN, Park DG, et al. Upregulation of stromal cell-derived factor 1alpha expression is associated with the resistance to neoadjuvant chemoradiotherapy of locally advanced rectal cancer: angiogenic markers of neoadjuvant chemoradiation. ONCOL REP. 2014;32『2493500 .

[17] Tokunaga R, Zhang W, Naseem M, Puccini A, Berger MD, Soni S, et al. CXCL9, CXCL10, CXCL11/CXCR3 axis for immune activation - A target for novel cancer therapy. CANCER TREAT REV. 2018;63凶40-7.

[18] Karin N, Razon H. Chemokines beyond chemo-attraction: CXCL10 and its significant role in cancer and autoimmunity. CYTOKINE. 2018.

[19] Chen E, Qin X, Peng K, Xu X, Li W, Cheng X, et al. Identification of Potential Therapeutic Targets Among CXC Chemokines in Breast Tumor Microenvironment Using Integrative Bioinformatics Analysis. CELL PHYSIOL BIOCHEM. 2018;45囚1731-46.

[20] Wu Y, Yuan L, Lu Q, Xu H, He X. Distinctive profiles of tumor-infiltrating immune cells and association with intensity of infiltration in colorectal cancer. ONCOL LETT. 2018;15ه3876-82.

[21] Bronger H, Karge A, Dreyer T, Zech D, Kraeft S, Avril S, et al. Induction of cathepsin B by the CXCR3 chemokines CXCL9 and CXCL10 in human breast cancer cells. ONCOL LETT. 2017;13『4224-30.

[22] Mandal G, Biswas S, Roy CS, Chatterjee A, Purohit S, Khamaru P, et al. Heterodimer formation by Oct4 and Smad3 differentially regulates epithelial-to-mesenchymal transition-associated factors in breast cancer progression. Biochim Biophys Acta. 2018;1864囚2053-66.

[23] Fang H, Jin J, Huang D, Yang F, Guan X. PAl-1 induces Src inhibitor resistance via CCL5 in HER2positive breast cancer cells. CANCER SCI. 2018.

[24] Fan L, Zhu Q, Liu L, Zhu C, Huang H, Lu S, et al. CXCL13 is androgen-responsive and involved in androgen induced prostate cancer cell migration and invasion. Oncotarget. 2017;8ه53244-61.

[25] Garg R, Blando JM, Perez CJ, Abba MC, Benavides F, Kazanietz MG. Protein Kinase C Epsilon Cooperates with PTEN Loss for Prostate Tumorigenesis through the CXCL13-CXCR5 Pathway. CELL REP. 2017;19ه375-88.

[26] Wei Y, Lin C, Li H, Xu Z, Wang J, Li R, et al. CXCL13 expression is prognostic and predictive for postoperative adjuvant chemotherapy benefit in patients with gastric cancer. Cancer Immunol Immunother. 2018;67ه261-9. 
[27] Singh SK, Mishra MK, Eltoum IA, Bae S, Jr. Lillard JW, Singh R. CCR5/CCL5 axis interaction promotes migratory and invasiveness of pancreatic cancer cells. Sci Rep. 2018;8囚1323.

[28] Allavena P, Sica A, Solinas G, Porta C, Mantovani A. The inflammatory micro-environment in tumor progression: the role of tumor-associated macrophages. Crit Rev Oncol Hematol. 2008;66®1-9.

[29] Zeelenberg IS, Ruuls-Van SL, Roos E. The chemokine receptor CXCR4 is required for outgrowth of colon carcinoma micrometastases. CANCER RES. 2003;63®3833-9.

[30] Desurmont T, Skrypek N, Duhamel A, Jonckheere N, Millet G, Leteurtre E, et al. Overexpression of chemokine receptor CXCR2 and ligand CXCL7 in liver metastases from colon cancer is correlated to shorter disease-free and overall survival. CANCER SCI. 2015;106ه262-9.

[31] Tang Z, Yu M, Miller F, Berk RS, Tromp G, Kosir MA. Increased invasion through basement membrane by CXCL7-transfected breast cells. AM J SURG. 2008;196®690-6.

[32] Yu M, Berk R, Kosir MA. CXCL7-Mediated Stimulation of Lymphangiogenic Factors VEGF-C, VEGF-D in Human Breast Cancer Cells. J ONCOL. 2010;2010®939407.

[33] Gautier L, Cope L, Bolstad BM, Irizarry RA. affy-analysis of Affymetrix GeneChip data at the probe level. BIOINFORMATICS. 2004;20®307-15.

[34] Irizarry RA, Hobbs B, Collin F, Beazer-Barclay YD, Antonellis KJ, Scherf U, et al. Exploration, normalization, and summaries of high density oligonucleotide array probe level data. BIOSTATISTICS. 2003;4囚249-64.

[35] Smyth GK. Linear models and empirical bayes methods for assessing differential expression in microarray experiments. Stat Appl Genet Mol Biol. 2004;3囚e3.

[36] Irizarry RA, Hobbs B, Collin F, Beazer-Barclay YD, Antonellis KJ, Scherf U, et al. Exploration, normalization, and summaries of high density oligonucleotide array probe level data. BIOSTATISTICS. 2003;4『249-64.

[37] Huang DW, Sherman BT, Tan Q, Kir J, Liu D, Bryant D, et al. DAVID Bioinformatics Resources: expanded annotation database and novel algorithms to better extract biology from large gene lists. NUCLEIC ACIDS RES. 2007;35『W169-75.

[38] Moriya Y, Itoh M, Okuda S, Yoshizawa AC, Kanehisa M. KAAS: an automatic genome annotation and pathway reconstruction server. NUCLEIC ACIDS RES. 2007;35『W182-5.

[39] Szklarczyk D, Franceschini A, Kuhn M, Simonovic M, Roth A, Minguez P, et al. The STRING database in 2011: functional interaction networks of proteins, globally integrated and scored. NUCLEIC ACIDS RES. 2011;39هD561-8. 
[40] Shannon P, Markiel A, Ozier O, Baliga NS, Wang JT, Ramage D, et al. Cytoscape: a software environment for integrated models of biomolecular interaction networks. GENOME RES. 2003;13₫2498504.

[41] Liu J, Lee J, Salazar HM, Mazitschek R, Ozcan U. Treatment of obesity with celastrol. CELL. 2015;161凶999-1011.

\section{Tables}

Table I. Gene Ontology terms enriched in the radiotherapy resistance module 


\begin{tabular}{|c|c|c|c|}
\hline ID & Term & Count & PValue \\
\hline GO:0071294 & Cellular response to zinc ion & 7 & 0.000000522 \\
\hline GO:0045926 & Negative regulation of growth & 6 & 0.0000132 \\
\hline GO:0071276 & Cellular response to cadmium ion & 5 & 0.000165 \\
\hline GO:0070062 & Extracellular exosome & 68 & 0.000916 \\
\hline GO:0000087 & CXCR3 chemokine receptor bindin & 3 & 0.00269 \\
\hline GO:0015893 & Drug transport & 3 & 0.002794 \\
\hline GO:0005102 & Receptor binding & 14 & 0.006603 \\
\hline GO:0005782 & Peroxisomal matrix & 5 & 0.006823 \\
\hline GO:0005777 & Peroxisome & 7 & 0.007474 \\
\hline GO:0000447 & $\begin{array}{l}\text { Endonucleolytic cleavage in ITS1 to separate SSU- } \\
\text { rRNA from 5.8S rRNA and LSU-rRNA from } \\
\text { tricistronic rRNA transcript (SSU-rRNA, } 5.8 \mathrm{~S} \\
\text { rRNA, LSU-rRNA) }\end{array}$ & 3 & 0.007563832 \\
\hline
\end{tabular}

Table II KEGG pathways enriched in the radiotherapy resistance module 


\begin{tabular}{|l|c|l|}
\hline \multicolumn{1}{|c|}{ Term } & Count & PValue \\
\hline Metabolic pathways & 37 & 0.0000000000173 \\
\hline & & \\
Mineral absorption & 8 & 0.0000000264 \\
\hline & & \\
Peroxisome & 7 & 0.00000804 \\
\hline & & \\
Purine metabolism & 8 & 0.000123 \\
\hline & & \\
Chemokine signaling pathway & 8 & 0.000183 \\
\hline & & \\
Pyrimidine metabolism & 6 & 0.000273 \\
\hline Glycine, serine and threonine metabolism & 4 & 0.000416 \\
\hline & & \\
Fatty acid degradation & 4 & 0.000582 \\
\hline & & \\
Fatty acid metabolism & 4 & 0.000789 \\
\hline Steroid hormone biosynthesis & & \\
\hline
\end{tabular}

Table III. The results of pathway analysis for the Connectivity Map database, $\mathrm{n}$ is the number of experiment, Percent non-null is the Effective experimental data. 


\begin{tabular}{|c|c|c|c|c|}
\hline Drug & enrichment & $\mathrm{n}$ & P-value & percent non-null \\
\hline isoxicam & -0.893 & 5 & 0.00004 & 100 \\
\hline adiphenine & -0.863 & 5 & 0.00008 & 100 \\
\hline diphenhydramine & -0.856 & 5 & 0.00012 & 100 \\
\hline pentoxifylline & -0.842 & 5 & 0.00026 & 100 \\
\hline Gly-His-Lys & -0.942 & 3 & 0.00028 & 100 \\
\hline thiamphenicol & -0.798 & 5 & 0.0007 & 80 \\
\hline & -0.787 & 5 & 0.00084 & 80 \\
\hline heptaminol & & & & \\
\hline
\end{tabular}

Figures 
A

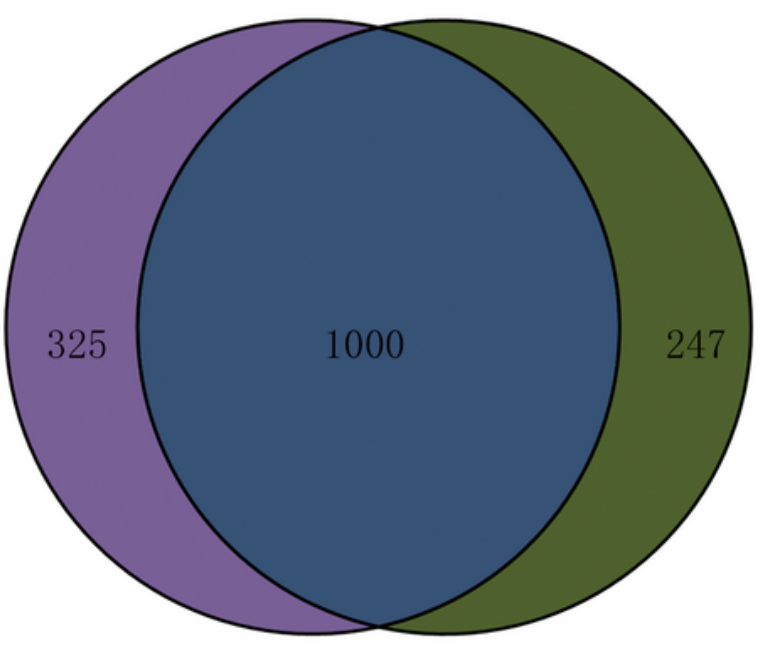

C

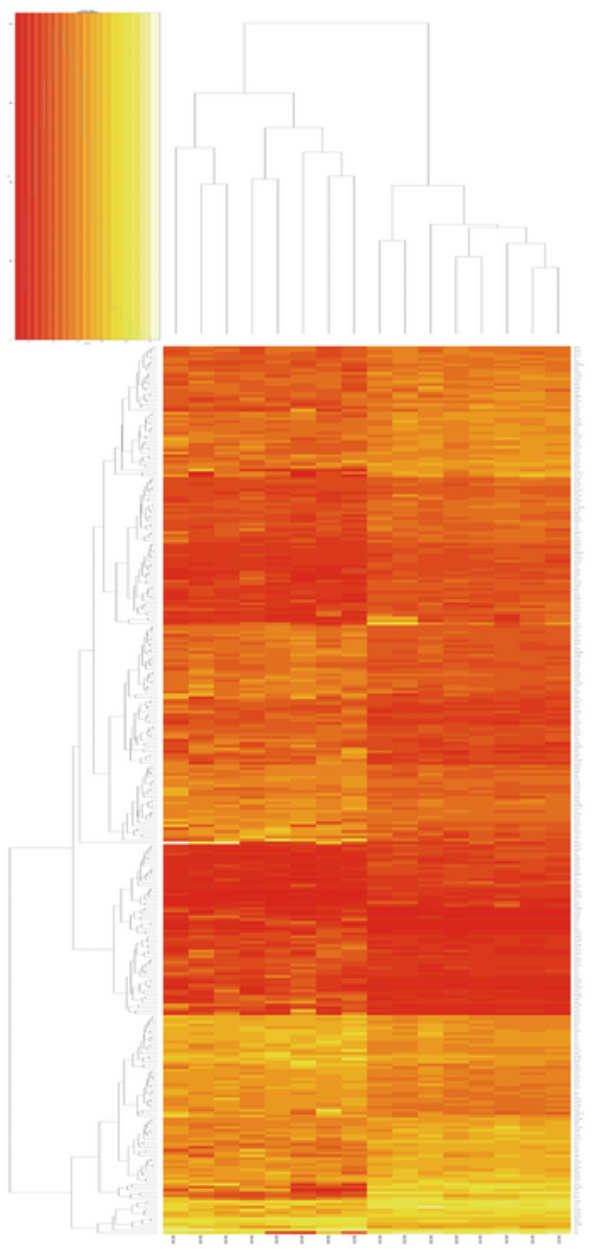

B

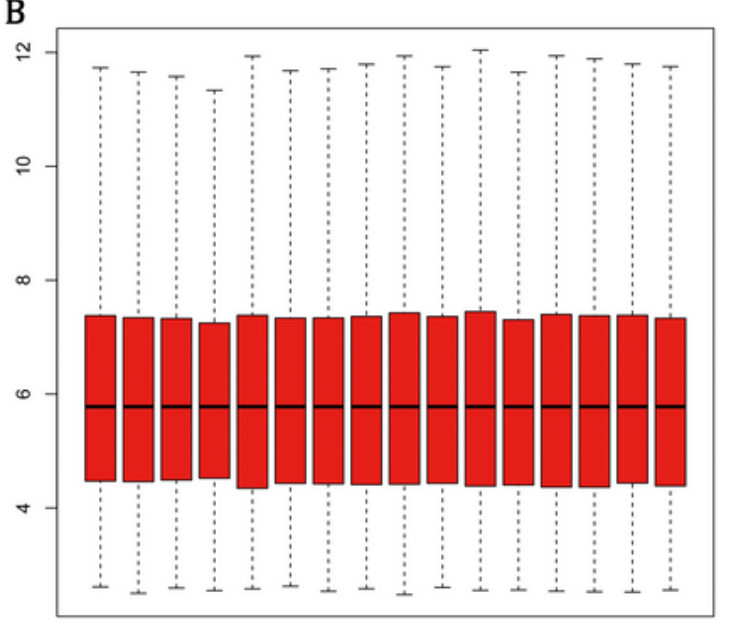

D

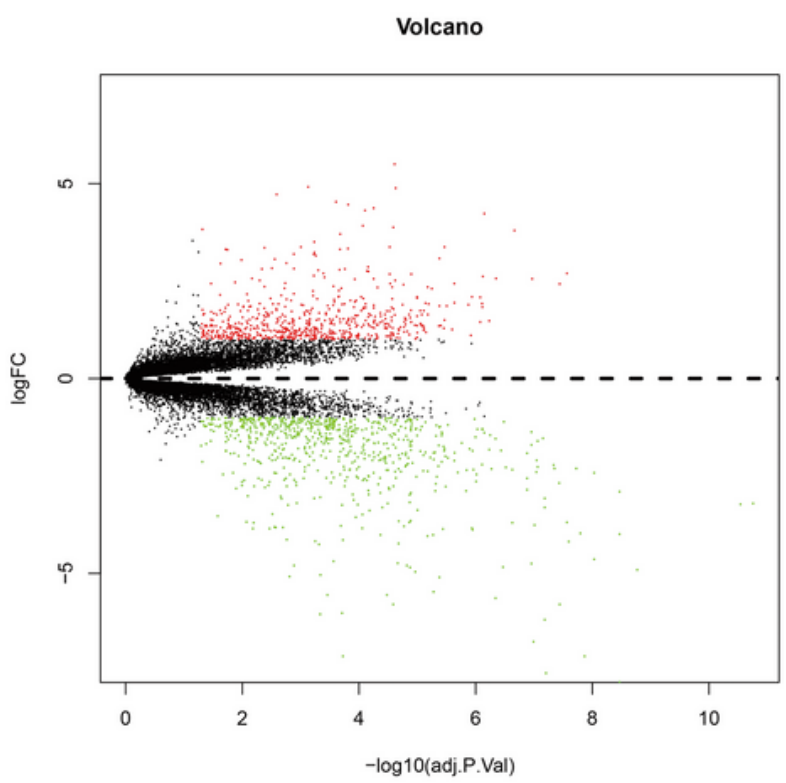

\section{Figure 1}

Identification of expression differences gene. (A) 1572 related genes are obtained. The 1000 genes in blue are considered to be common in rectal cancer tissues. 325 in purple are unique genes in the radiotherapy-resistance rectal tumor group. 247 in green are unique genes in the radiotherapy-sensitive rectal tumor group, which are also considered to be unique differential gene in the sensitive group. (B) The normalized boxplot. The original data were normalized by R package limma. (C) The heat map 
shows expression patterns of different samples. The horizontal axis represents the sample name. The left vertical axis shows a set of DEGs and the upper horizontal axis a series of samples. Yellow and red represents the up-regulated and the down-regulated genes respectively. (D) Volcano plot of the differential gene expression analysis. Red dots with log fold change $\geq 1$, green dots with log fold change $\leq-1 P$-value $<0.05$.

A

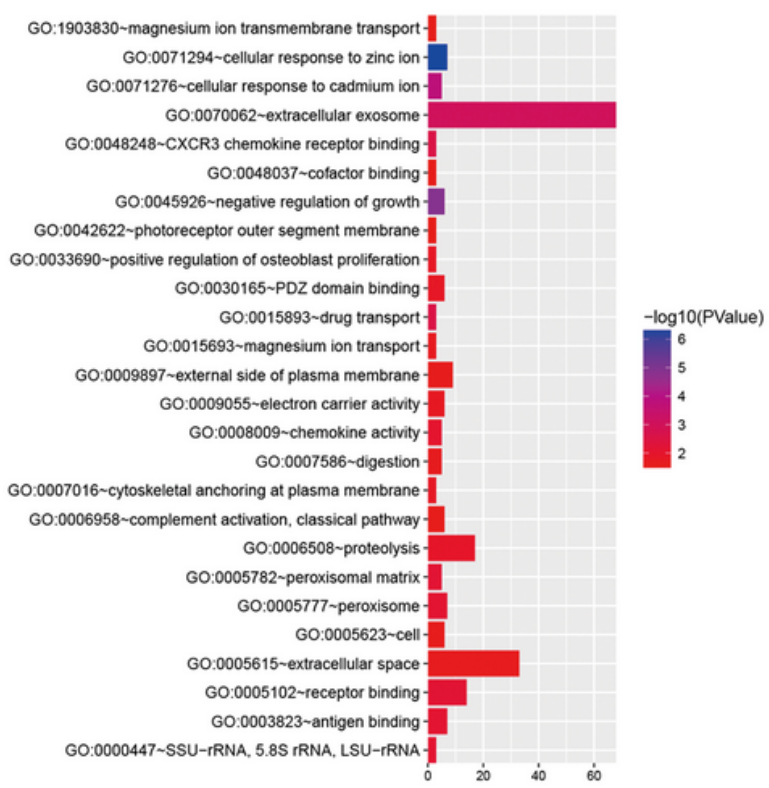

C

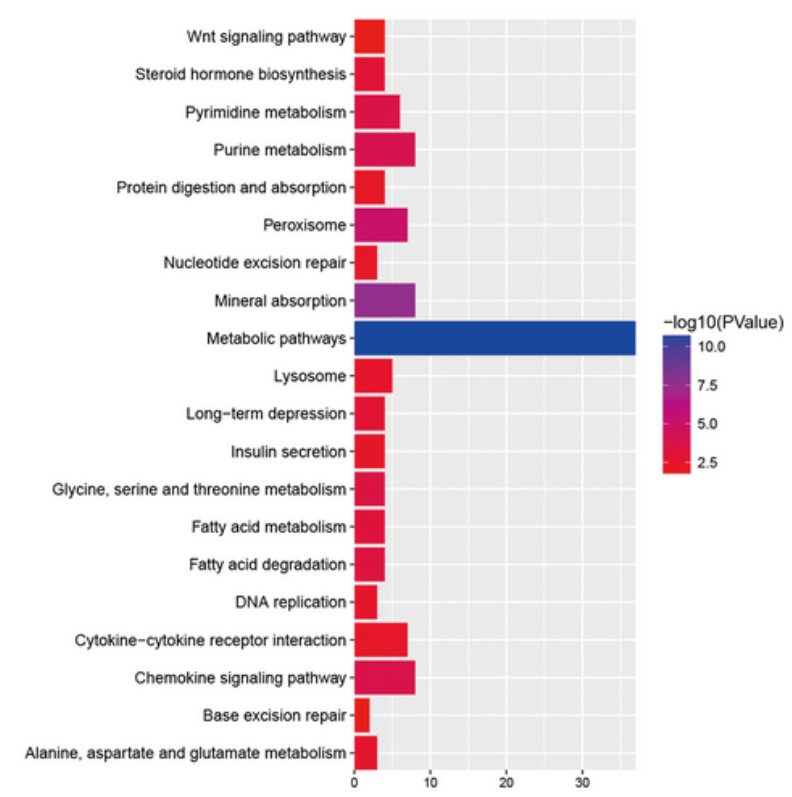

B

Biological process Cellular component Molecular function

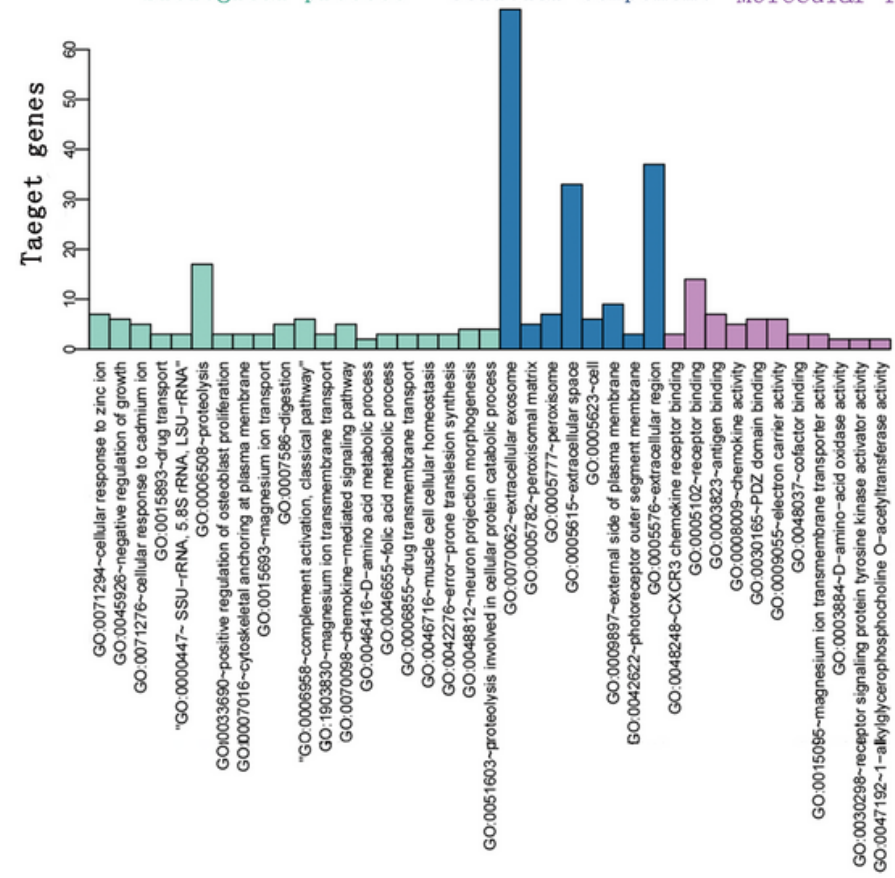

Figure 2 
GO terms and enriched KEGG pathway of functional enrichment analysis. (A) 20 enriched gene ontology for differentially expressed genes. Gene count of cellular response to zinc ion, negative regulation of growth, cellular response to cadmium ion, extracellular exosome, CXCR3 chemokine receptor binding, drug transport, receptor binding, peroxisomal matrix, peroxisome, antigen binding, chemokine activity, proteolysis, positive regulation of osteoblast proliferation, cytoskeletal anchoring at plasma membrane, PDZ domain binding, electron carrier activity, magnesium ion transport, digestion, extracellular space. (B) The green is biological process. The blue is cellular component. The purple is molecular unction. The Yaxis is the number of gene $(P<0.05)$. (C) 20 pathways enriched KEGG pathway. Gene count of Metabolic pathways, Mineral absorption, Peroxisome, Purine metabolism, Chemokine signaling pathway, Pyrimidine metabolism, Glycine, serine and threonine metabolism, Fatty acid degradation, Wnt signaling pathway, Fatty acid metabolism, Steroid hormone biosynthesis, Long-term depression, DNA replication, Alanine, aspartate and glutamate metabolism, Lysosome, Base excision repair, Insulin secretion, Cytokine-cytokine receptor interaction, Protein digestion and absorption, Nucleotide excision repair. 
A
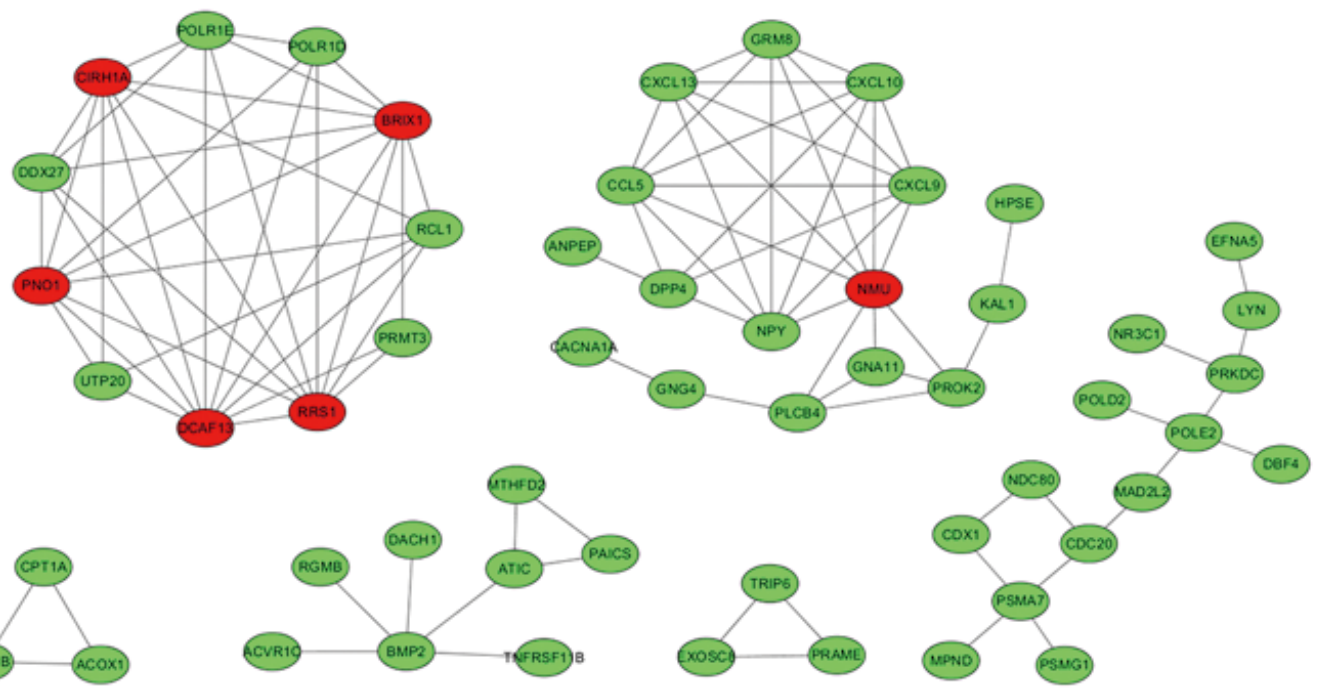

B
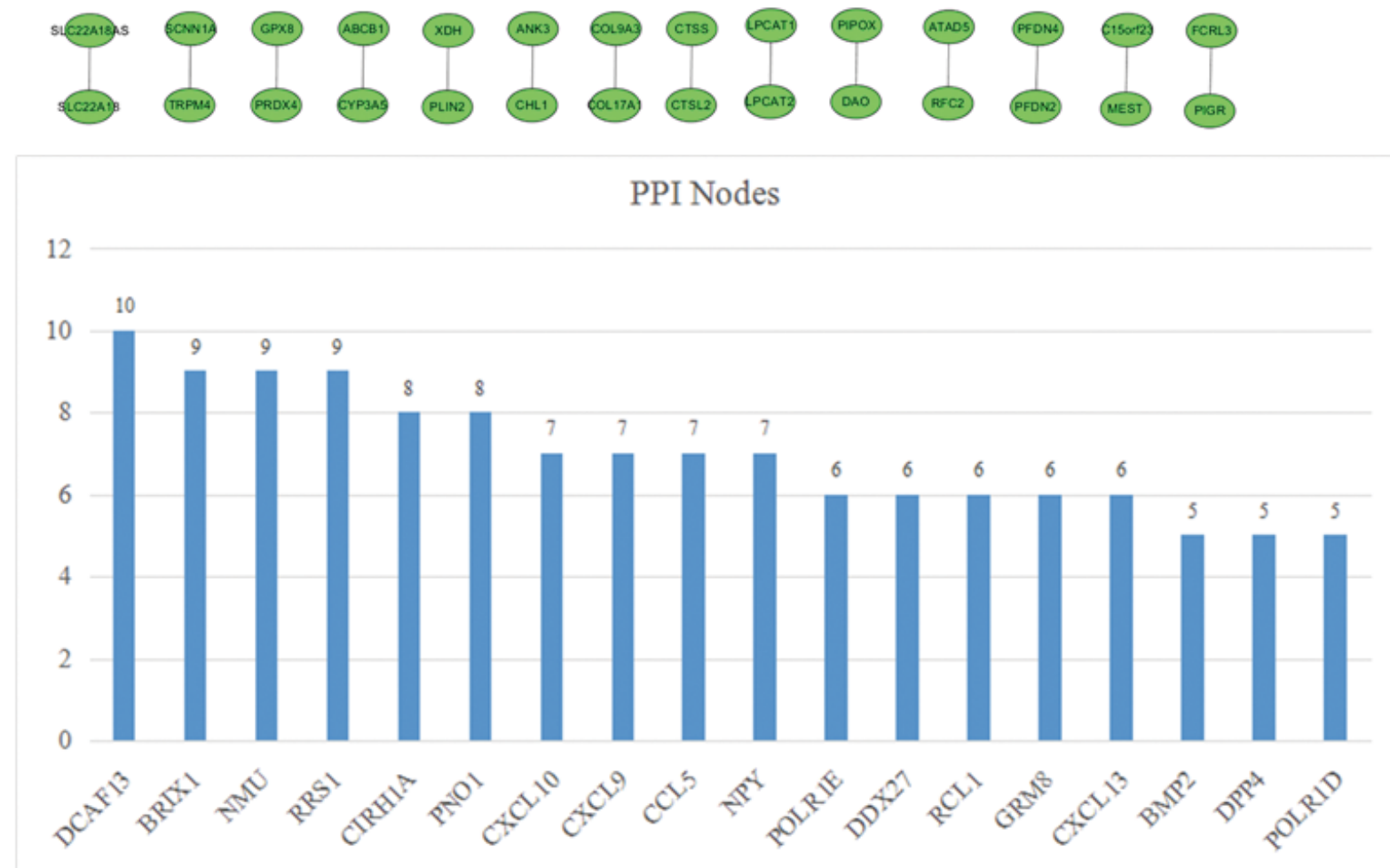

\section{Figure 3}

Protein-protein interaction (PPI) networks of differentially expressed genes (DEGs) (A) PPI network showing DEGs by Cytoscape from STRING database, Nodes stand for proteins and edges represent interactions between two proteins. (B) Histogram statistics for node distribution in PPI. 
A

B

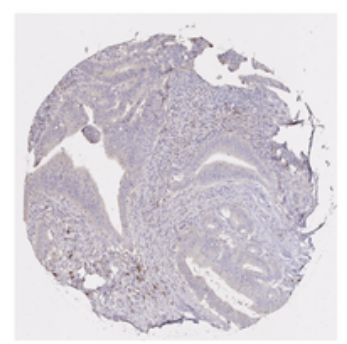

Normal

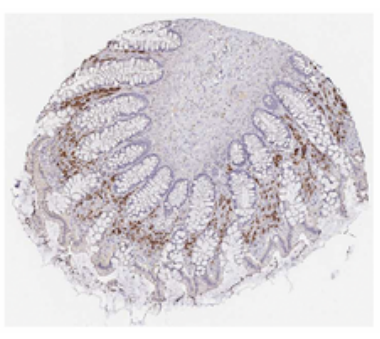

Tumor

CXCL10

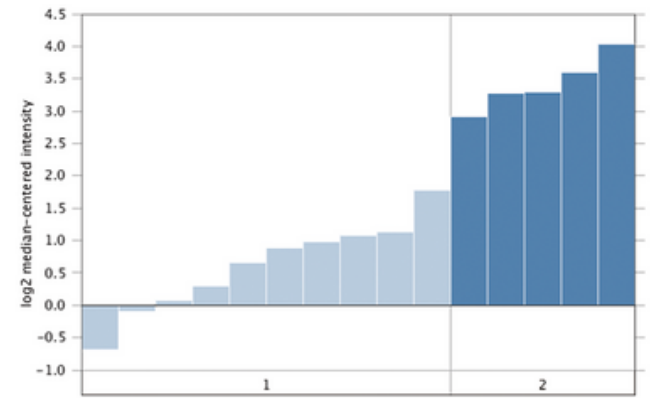

C
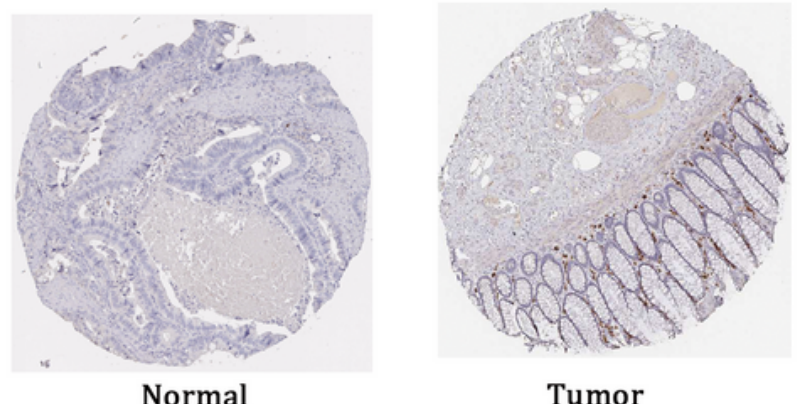

Tumor

CXCL13

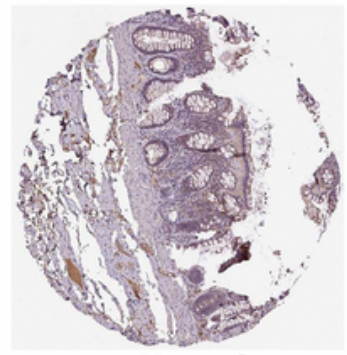

Normal

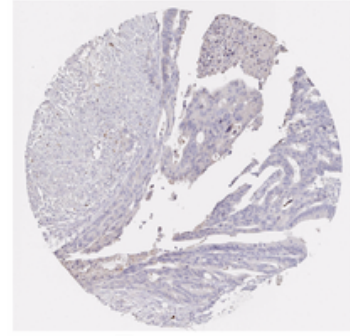

Tumor

CCL5

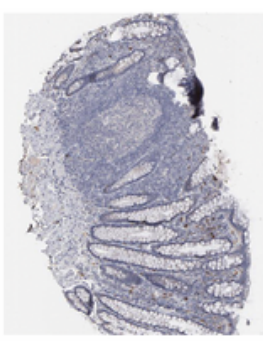

Normal
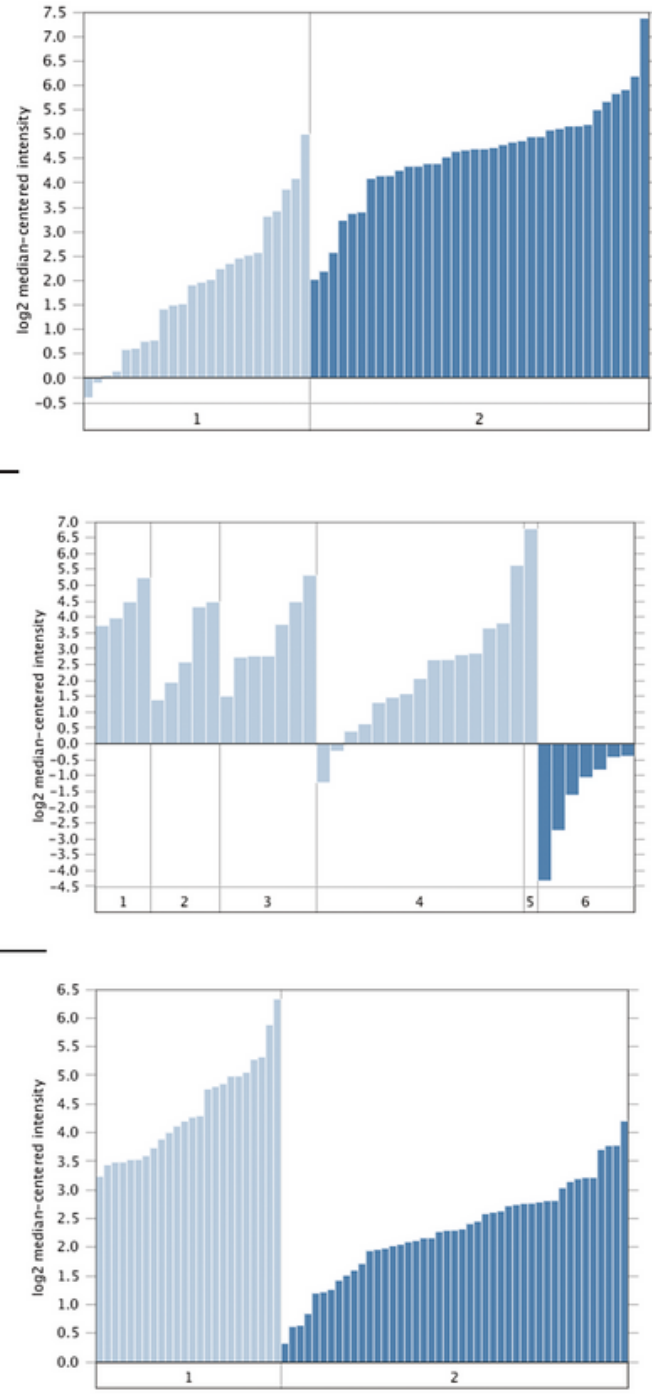

\section{Figure 4}

Gene expression in human rectal cancer specimens. Four genes expression in normal rectal tissue and rectal cancer specimens. Images were taken from the Human Protein Atlas (http://www.proteinatlas.org) online database (left). Oncomine data showing gene expression in normal vs tumor of recal (right). Figure 5 right, the $Y$ axis is the expression value of gene in sample. (A right) CXCL9: $X$ axis label 1 is normal of rectal tissue. $\mathrm{X}$ axis label 2 is tumor of reatal tissue. (B right) CXCL10: $\mathrm{X}$ axis label 1 is normal of rectal 
tissue. $X$ axis label 2 is tumor of reatal tissue. (C right) CXCL13: $X$ axis label 1 is normal of ascending colon. $X$ axis label 2 is normal of descend colon. $X$ axis label 3 is normal of reatal. $X$ axis label 4 is normal of transverse colon, $X$ axis label 5 is normal of sigmoid colon, $X$ axis label 6 is tumor of colorectal. (D right) CCL5: $X$ axis label 1 is normal of rectal tissue. $X$ axis label 2 is tumor of reatal tissue. 